\title{
Characterization of Complex Generic Drug Products Using Cryogenic Electron Microscopy
}

Jiwen Zheng

US FDA, Silver Spring, Maryland, United States

A variety of complex drug formulations including liposomes, emulsions, suspensions, micelles, polymeric nanoparticles, drug-protein complexes, iron complexes have been developed to enhance the bioavailability of active pharmaceutical ingredients (APIs). The complex nature of these formulations presents significant challenges to the development and approval of generic drug products.

It has been reported that subtle variations in manufacturing processes can cause noticeable differences in physicochemical properties (e.g., particle size and morphology) of drug products that are formulated qualitatively (Q1) and quantitatively (Q2) the same. These differences may ultimately impact the drug release behavior and bioavailability of APIs. Therefore, the equivalence of proposed generic complex drug products to the reference listed drugs (RLDs) is in part established by evaluating the product's physiochemical properties such as particle size and morphology. Techniques such as laser diffraction, dynamic light scattering, and nanoparticle tracking analysis are useful in determining particle size and size distribution. However, none of these techniques can evaluate particle morphology, which critically affects drug performance and release.

In order to fulfill this demand on demonstrating comparable size and morphology between generic formulations and RLD, reliable and suitable characterization techniques need to be identified and carefully assessed. In our studies, a wide variety of complex drug products including intravenous iron injections, ophthalmic emulsions, anesthetic emulsion/liposome injections, anticancer liposomal injections and protein-bound paclitaxel nanoparticles were investigated using Cryo-EM $(1,2,3,4)$. Our findings reveal the importance of cryo-EM technique in appropriate estimation of the morphology of the drug products in comparison to traditional room temperature EM method. Cryo-EM may become a prospective technique to support a demonstration of drug product sameness.

\section{References}

[1] Y Wu, P Petrochenko, L Chen, S Wong, M Absar, S Choi, J Zheng, Int. J. Pharm. 2016, 505,167-74.

[2] PE Petrochenko, N Pavurala, Y. Wu, W Yee, H Parhiz, K Chen, SM Patil, H Qu, Buoniconti P, Muhammad, S Choi, D Kozak, M Ashraf, CN Cruz, J Zheng, X Xu. Int. J. Pharm. 2018, 550, 229-239.

[3] S Manna, Y Wu, Y Wang, B Koo, L Chen, P Petrochenko, Y Dong, S Choi, D Kozak, B Oktem, X $\mathrm{Xu}$, J Zheng, J Control Release. 2019, 294, 279-287.

(4) Y Wu, S Manna, P Petrochenko, B Koo, X Xu, S Choi, D Kozak, J Zheng, Int. J. Pharm. 2020, 577, 118998. 